\title{
Social media live streaming y su impacto en los medios de comunicación: El caso de YouTube Live
}

\author{
Alexis Apablaza-Campos \\ Universidad de las Artes, Ciencias y Comunicación, UNIACC y Universitat Pompeu Fabra \\ a_apablaza@boleteador.com \\ https://orcid.org/0000-0003-4407-1145
}

\section{Social media live streaming and its impact on the news media: the case of YouTube Live}

\begin{abstract}
RESUMEN
Las redes sociales de vídeo en directo, comprendidas como Social Media Live Streaming (SMLS), son una representación de una forma emergente de comunicación audiovisual interactiva en el contexto de los medios de comunicación. EL SMLS puede presentarse en tres formas (redes sociales propias, funcionalidades de perfiles y aplicaciones externas). YouTube, que en 2008 realizó su primera emisión en directo y una década más tarde abrió la funcionalidad a todos sus usuarios, experimentó con cada una de esas formas. Actualmente, YouTube Live (como funcionalidad de canales) es el principal formato SMLS de la red social perteneciente a Google. Diversos editores de contenidos han experimentado un importante aumento de sus resultados por sobre otras redes de live streaming.

El siguiente artículo presenta una revisión sistematizada sobre las emisiones Social Media Live Streaming a través de YouTube Live y un estudio de caso sobre su uso en medios televisivos de Venezuela durante las Elecciones Presidenciales 2018.
\end{abstract}

PALABRAS CLAVE

Social Media Live Streaming, SMLS, YouTube, YouTube Live, Medios digitales, Venezuela

\section{ABSTRACT}

Social Media Live Streaming (SMLS) is an emerging form of interactive audio-visual communication in the digital context. The SMLS can be presented in three ways: as a social network exclusively devoted to SMLS, as one of the functionalities of the network, and as an external application. YouTube made its first live broadcast in 2008, and a decade later opened the functionality to all users, experimenting with each of these latter ways.

Currently, YouTube Live (as a channel functionality) is the main SMLS format of the social network. Several content publishers have experienced a significant increase in their results over other live streaming networks.

This article presents an analysis design of Social Media Live Streaming broadcasts through YouTube Live and its application in the case of Venezuelan television media during the 2018 Presidential Elections.

\section{KEY WORKS}

Social Media Live Streaming, SMLS, YouTube, YouTube Live, digital news media, Venezuela 


\section{Introducción}

En línea con el sugerente lema de este número, creemos que es posible sostener que "el futuro digital de los hechos" pasa por el vídeo. Existen diversos análisis que comparten esta predicción (Kalogeropoulos, Cherubini, \& Newman, 2016) (Argila, 2017) (Castillo, 2017), sostenida mediante tres grandes premisas de fácil distinción en los entornos digitales de hoy:

1. Crecimiento continuo en velocidades de conexión tanto en instalaciones fijas (red y WiFi) como en accesos móviles (4G y 5G) (Villas-Boas, 2018).

2. Mayor capacidad para la emisión y/o recepción de contenidos audiovisuales debido a la presencia en el mercado de dispositivos móviles con mayor capacidad de procesamiento (El Economista América, 2016).

3. Creciente consumo de contenidos audiovisuales a través de apps, redes sociales y/o plataformas de streaming (McCue, 2017).

Dentro de este escenario es totalmente comprensible la aparición de webs y apps dedicadas a la emisión/reproducción de vídeos en directo. Sin embargo, los fallidos casos de Justin.tv ${ }^{1}$ y de Meerkat ${ }^{2}$ clarifican que la llegada del Social Media Live Streaming (SMLS) va más allá de ser un simple software que provea contenidos multimedia en directo.

Trabajos anteriores (Apablaza-Campos, 2017a) nos han permitido categorizar como SMLS a las tecnologías que cumplan con el siguiente doble funcional:

1. Producción y distribución de vídeos en directo, en diferentes formatos, a través de las redes sociales, mediante aplicaciones propias y/o asociadas.

2. Entrega al emisor de feedback instantáneo: número de usuarios conectados, mensajes y reacciones de sus audiencias.

Actualmente, el SMLS funciona mediante redes sociales propias (Periscope), funcionalidades para perfiles (Facebook Live), y aplicaciones externas (Kanvas, YouNow, Upclose). Curiosamente YouTube -que comenzó a experimentar con emisiones en directo desde hace más de una década- ha probado cada una de estas opciones hasta quedarse con la segunda solo desde hace algunos meses.

\section{Presentación y componentes}

A continuación, y una vez presentado el contexto en el que se inscribe esta investigación, se detallan sus diversos componentes antes de pasar a discutir sus resultados.

\subsection{Marco teórico}

La investigación presentada en este trabajo utiliza un marco teórico compuesto por tres líneas principales:

1. Periodística (Teoría del Periodismo): estudio de características y procesos que determinan la producción de contenidos de actualidad, entendiendo el consumo de medios de comunicación como "un círculo de realidad envolvente que se convierte en referencia diaria de nuestra vida" (Gomis, 1991, pág. 13). En este apartado, también consideramos las Newsonomics como un conjunto de leyes que rigen tanto la selección de contenidos informativos como los resultados financieros de las empresas periodísticas (Doctor, 2010).

2. Ecología de los Medios: siguiendo la influencia de Marshall McLuhan, Neil Postman desarrolló esta teoría que permite analizar los medios de comunicación dentro de su contexto global. "Ningún medio adquiere sentido o existencia de manera aislada, sino en constante interrelación con otros medios" (Scolari, 2015, pág. 18).

3. Cibermedios: medios de comunicación digitales que cuentan con la multimedialidad, hipertextualidad e interactividad como principales características. Definidos como "sitios web cuya finalidad esencial es el desarrollo y transmisión de contenidos informativos, esto es, aquellos sitios web que cumplen en internet el papel de los medios de comunicación social" (Palacios \& Díaz Noci, 2009, pág. 21).

\subsection{Marco metodológico}

En primer lugar, para el presente trabajo se llevó a cabo una revisión sistematizada sobre las publicaciones más recientes vinculadas con el tema central, con el fin de establecer el contexto de YouTube Live como SMLS y que pueden consultarse en la bibliografía.

A partir de lo anterior, se realizaron diversos análisis comparativos que nos permitieron iniciar una línea de propuestas entre las que se encuentran el diseño desarrollado para este estudio.

En tercer lugar, implementamos un análisis heurístico para analizar perfiles de SMLS determinados, aplicando una serie de indicadores (KPI) para medir la efectividad de estos canales.

\subsubsection{Estudio de caso}

Todo este contexto permitió la elaboración de un estudio de caso (Yin, 2018), cuya aplicación ha permitido la obtención de los resultados que se analizarán en las líneas siguientes. 
A continuación, se presentan los detalles del estudio de caso de medios venezolanos analizados en este trabajo:

- Fecha: 20 de mayo de 2018 .

- Medios: Venezolanos por la Información, Venevisión, Globovisión, TeleSUR, Televen y Venezolana de Televisión.

- SMLS: YouTube Live.

- Total de emisiones: 6

La fecha elegida corresponde a la realización en Venezuela de las elecciones de tipo presidencial y legislativa para el período 2019-2025, en el caso del primero, y 2018-2021, en el caso del segundo.

Sobre los medios seleccionados para el estudio es importante destacar:

1. Venevisión: fundado en 1961 es la estación televisiva más antigua de Venezuela y habitual líder en sintonía. Su canal informativo en YouTube se llama "Noticiero Venevisión" y cuenta con cerca de 110 mil seguidores.

2. Televen: fundado en 1988 es un medio privado generalista y principal competidor de Venevisión. Tiene dos canales en YouTube: "Televen TV" y "Televen EN VIVO", este último está dedicado exclusivamente a las emisiones en directo y tiene más de 25 mil seguidores.

3. Globovisión: fundado en 1994 es un canal privado de contenidos informativos en formato 24 horas. Al igual que Televen, en YouTube dispone de dos canales: "Globovisión Vídeos" y "Globovisión en directo", este último supera los 40 mil suscriptores.

4. TeleSUR: medio de televisión público perteneciente a los gobiernos de Venezuela, Cuba, Bolivia, Nicaragua y Uruguay, con sede en Caracas y fundado en 2005. En YouTube cuenta con canales en castellano (TeleSUR TV), inglés (TeleSur English) y portugués (TeleSUR PT). La primera de ellas supera los 300 mil suscriptores.

5. Venezolana de Televisión: cadena de televisión pública fundada en 1964. Para realizar su cobertura informativa del proceso eleccionario vía YouTube, creó un canal llamado "VTV Voto 2018 - El pueblo decide" con cerca de 200 suscriptores.

6. Venezolanos por la Información: canal de TV vía streaming creado en 2016 con contenidos informativos. Su canal en YouTube se llama "VPItv", desde donde realiza emisiones en directo en formato $24 / 7$, y cuenta con cerca de 230 mil suscriptores.

\subsection{Objeto de estudio y objetivos}

El objeto de estudio de este trabajo corresponde a las emisiones SMLS de medios de comunicación venezolanos en YouTube Live con ocasión de la cobertura de las Elecciones Presidenciales 2018.

El objetivo principal es explorar a YouTube Live como una funcionalidad Social Media Live Streaming para canales de YouTube, buscando comprender su papel a nivel de innovación y tendencias para el periodismo digital.

Los objetivos secundarios son los siguientes:

- Comprender la integración de YouTube Live como herramienta de distribución de información y su uso en las coberturas periodísticas.

- Analizar impactos y resultados de medios digitales que utilizan la red como su principal canal de distribución y emisión de videos en directo.

- Describir los impactos y resultados de cibermedios venezolanos mediante un estudio de caso.

\section{Antecedentes}

\section{1 ¿Es YouTube una red social?}

Una rápida revisión de estadísticas recientes permite comprender el alcance y la magnitud de YouTube, la segunda web con más tráfico en el mundo (Alexa, 2018) solo superada por su matriz Google:

- Es usado por una cuarta parte de la población mundial (PuroMarketing, 2018).

- Más de mil millones de usuarios, su target es de 18 a 34 años, disponible en 88 países y en 76 idiomas, y cuenta con más de mil millones de horas de reproducción diarias (YouTube, 2018a).

- $\quad$ Es la red social más usada en los Estados Unidos (78\%) (Smith \& Anderson, 2018).

- $\quad$ En el caso de los adolescentes norteamericanos (de 13 a 17 años), la cifra crece en un 85\% (BBC News Mundo, 2018).

No obstante, ¿podemos considerar a YouTube como una red social propiamente tal o bien se debe categorizar exclusivamente como una plataforma de reproducción de vídeos online? Antes de buscar una respuesta, consideramos necesario revisar las principales definiciones de qué es una red social para establecer sus alcances:

- Un sitio en la red cuya finalidad es permitir a los usuarios 
relacionarse, comunicarse, compartir contenido y crear comunidades (ONTSI, 2011).

- Servicios dentro de las webs que permiten al usuario:

- Construir un perfil público o semi-público dentro de un sistema limitado

- Articular una lista de otros usuarios con los que comparte una conexión

- Visualizar y rastrear su lista de contactos y las elaboradas por otros usuarios dentro del sistema

La naturaleza y nomenclatura de estas conexiones suele variar de una red social a otra (Boyd \& Ellison, 2007).

Todo lo referenciado en el primer punto es posible de encontrar en YouTube, tanto sus vídeos, mensajes de usuarios en las publicaciones, como sus canales y sugerencias del news feed permiten la relación, comunicación, compartir contenido y creación de comunidades mencionadas en una de las definiciones que más consenso genera tanto en académicos como en expertos digitales.

El segundo punto, vinculado mayormente a las conexiones entre usuarios, es aplicable a los canales de cada usuario, creados por YouTube a cada usuario independiente de si ha publicado vídeos. Si bien es cierto que solo los propietarios del canal pueden conocer la identidad de sus seguidores, existe la opción de configurar la privacidad de publicaciones y de otras acciones (como favoritos y/o listas de reproducción) además de la opción de seguir canales con contenido relacionado al perfil que se está visitando.

Si en ambos casos, podemos concluir que la categorización como red social de YouTube es afirmativa, entonces ¿por qué merece punto de discusión (y/o aclaración)? Esto se debe a dos factores principales:

1. Aunque su creación coincide las primeras redes sociales (hi5 en 2002, Myspace en 2003, YouTube y Facebook en 2004, Twitter y Tuenti en 2006), en un comienzo destacó más por su manera de revolucionar la generación y el consumo de vídeos en línea (Nación, 2006) que por el intercambio de contenido personal entre amigos como sucedió con Facebook y MySpace.

2. La categorización de los usuarios como "videoteca digital" y no como "red social". Un ejemplo de ello sucedió en el II Estudio de Redes Sociales en Internet de IAB Spain, cuando solo un 3\% de los encuestados reconoció espontáneamente a YouTube como red social; en cambio, cuando se les preguntó si la conocían un 85\% respondió de manera afirmativa y un 60\% dijo usarla frecuentemente (IAB Spain, 2010).

Con los antecedentes debidamente analizados, es posible ir más allá e intentar comprender si YouTube Live efectivamente cumple con el doble funcional mencionado en el subapartado anterior sobre las tecnologías SMLS. Mientras por un lado permite la producción y distribución de vídeos en directo mediante una aplicación que cumple con los requisitos para ser considerada como una red social propiamente tal; por otro, el emisor del contenido puede acceder a la información constantemente actualizada sobre reproducciones, chat en vivo y reacciones ("Me gusta" y "No me gusta").

\subsection{SMLSy YouTube Live}

Es innegable que tanto YouTube, como plataforma de vídeos online, como Netflix, como plataforma de streaming, han propiciado un consumo mayoritario de contenidos audiovisuales desde finales de la década pasada, estimulando el posterior crecimiento y desarrollo del live streaming.

En trabajos anteriores (Apablaza-Campos, 2017c), hemos explicado que desde el nacimiento de Periscope (2015) en adelante es posible hablar del Social Media Live Streaming. Sin embargo, aquí es necesario establecer diferencias con lo sucedido con YouTube debido a que su primera emisión en directo se realizó en 2008, pero se demoró una década (marzo de 2018) en permitir que todo usuario con un canal en la red pudiese emitir desde su PC o móvil (Ong, 2018).

Para comprender mejor los continuos cambios de la red de vídeos de Google y su evolución en el SMLS, se ha construido la siguiente línea temporal:

- 2008: nace YouTube Live, pero con un fin totalmente distinto al actual. Consistía en un evento realizado en simultáneo en Estados Unidos y Japón con la participación de artistas y los primeros youtubers conocidos que se emitía en vivo a través de la plataforma (Dobuzinskis, 2008).

- 2009-2010: a través de aplicaciones externas, y mediante acuerdos comerciales, se realizan dos emisiones de prueba, un concierto de U2 y una sesión de preguntas y respuestas con el presidente Barack Obama (Gross, 2010). También se realizan pruebas con infraestructura propia.

- 2011: YouTube Live deja atrás los conciertos y se anuncia oficialmente como un espacio de creación de emisiones en directo limitada solo a socios seleccionados (YouTube, 2011).

- 2012: emisión en tiempo real de grandes eventos como los Juegos Olímpicos de Londres, el festival Lollapalooza y el lanzamiento desde la estratósfera del austriaco Felix Baumgartner.

- 2013: las emisiones en directo están disponibles para todos los usuarios con cuenta verificada por la red social (MacNN, 2013). 


\section{Emite en directo sin importar el momento o el motivo.}

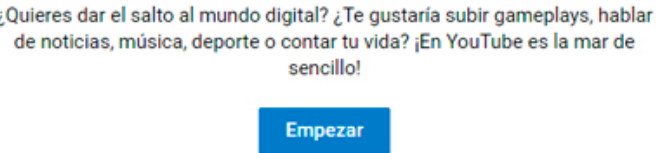

Empezar

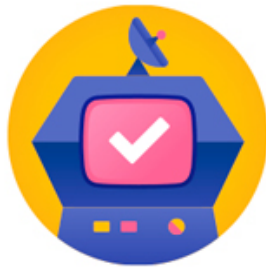

Vayamos por partes.

Comprobaremos que tu cuenta está en buen estado y verificaremos tu identidad a través de un número de teléfono. Pueden pasar 24 horas antes de que tengas acceso.

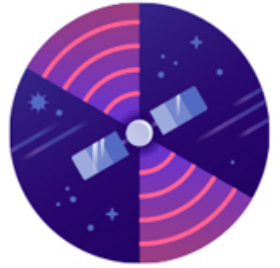

¿A qué esperas para empezar con la emisión?

Pondremos a tu disposición la URL youtube.com/channel/UCYCaeyz7GGcJ5QAicbgtnBA/live para que promociones tus emisiones y tus fans puedan localizarte sin problema.

Figura 1. Mensaje de habilitación de YouTube Live (YouTube, 2018b).

- 2016: debido al crecimiento de Periscope y Facebook Live se rumorea que se lanzará una red social propia de SMLS llamada YouTube Connect (Yeung, 2016). Sin embargo, se decide a partir de junio potenciar YouTube Live con YouTube Live Events que permite emisiones en directo a todos quienes habiliten la funcionalidad y dispongan de un decodificador (Adveischool, 2016b). Además, los youtubers disponen de YouTube Community, una pestaña adicional en sus canales donde es posible encontrar su historial de vídeos en directo (Adveischool, 2016a).

- 2017: Lanzamiento de la versión móvil de YouTube Live solo para cuentas verificadas, permitiendo también vídeos en calidad $4 \mathrm{~K}$ y en formato mixed reality (inmersivos, realidad aumentada y realidad virtual). También se presentó una versión llamada Super Chat para realizar donaciones a los emisores y una aplicación para dar soporte de vídeos en directo a webs externas (Parker, 2017).

- 2018: todos los canales de YouTube pueden realizar emisiones en directo desde computadoras o móviles, además del lanzamiento de las siguientes características adicionales: subtítulos automáticos, chat en directo que puede conservarse después de la emisión y etiquetas de geolocalización por parte del emisor (Chowdhry, 2018).
Para emitir en directo a través de YouTube, es necesario habilitar la funcionalidad antes de la primera transmisión y esperar un mínimo de 24 horas para que la red social verifique la información del propietario del canal y le permita crear contenido en alguna de sus dos modalidades de emisión (ver figura 1 y figura 2):

- YouTube Live: vídeos en directo para usuarios convencionales. Recomendado para quienes desean contar sus experiencias frente a la cámara o bien emitir sucesos de manera espontánea.

- YouTube Live Events: emisiones profesionales conectando diversas cámaras y controlando sus niveles de audio respectivos. Recomendado para conciertos, eventos deportivos y debates.

\section{YouTube Live y los medios de comunicación}

\subsection{El valor para los editores de contenidos}

En abril de 2017, Fidji Simo, jefa de vídeos de Facebook, anunció que Live se había convertido en la red de SMLS con mayor número de usuarios y que uno de cada cinco contenidos

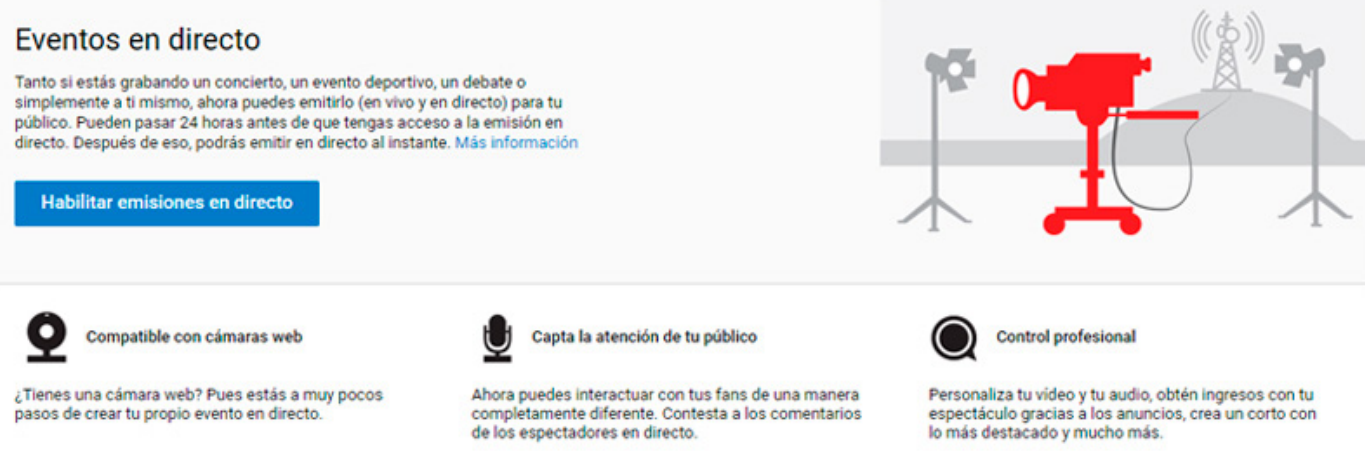

Figura 2. Mensaje de habilitación de YouTuve Live Events (YouTube, 2018c). 
audiovisuales reproducidos en su red social correspondían a emisiones en directo (Constine, 2017). Gran parte de ello se debió a un programa de subsidios aplicado a editores, marcas e influencers asegurando la exclusividad en los contenidos (Perlberg \& Sheetharaman, 2016).

Sin embargo, para fines de 2017 los pagos comenzaron a disminuir en unos y se terminaron para otros. Ello, sumado al cambio de algoritmo de Facebook aplicado desde principios de enero (Zuckerberg, 2018), llevó a que el Columbia Journalism Review del Tow Center publicara en febrero de 2018 un reporte llamado "RIP Facebook Live", afirmando que el volumen de publicaciones en directo de los medios que recibieron el estímulo económico cayó en un 94\% (Brown, 2018).

Esta situación, sumados a los escándalos de filtración de datos, tienen a la red social tratando de moverse en aguas revueltas, con usuarios de todo el mundo cerrando perfiles y grandes medios como Folha de São Paulo o Playboy anunciado el fin de la generación de contenido e incluso la eliminación total de sus fanpages (Coldewey, 2018).

Como hemos podido observar durante los primeros meses de 2018, una estrategia de salida o de disminución en la importancia de un canal social no necesariamente implica una desinversión de tiempo y recursos para RRSS en general, sino bien es un cambio de prioridades de los perfiles existentes.

Concretamente en el caso del SMLS, Twitch -que tomó el relevo de Justin.tv y hoy es propiedad de Amazon- está comenzando a dar un giro en su servicio de live streaming para videojuegos y ha comenzado a recibir contenidos en directo de medios digitales como BuzzFeed (Southern, 2018). Sin embargo, diversos editores coinciden que la tecnología SMLS que más ha crecido en cuanto a alcance y resultados es YouTube Live.

Un estudio realizado por Digiday UK a diversos medios estadounidenses -la mayoría de ellos con años de emisiones SMLS a través de la red social de Google- permite comprobar una importante mejora en sus resultados durante 2018 sin haber realizado grandes cambios en cuanto a contenidos y formato de emisiones de años anteriores (Peterson, 2018b):

- Time: el equipo digital de la prestigiosa revista perteneciente a Meredith realiza el $90 \%$ de sus emisiones SMLS en simulcasting a través de YouTube Live y de Facebook Live, reconociendo que sus resultados son "significativamente mayores" en el primero de ellos. En 2017, Time emitió un total de 1.600 horas de vídeo en directo a través de YouTube y, según han comprobado, el volumen de visualizaciones es 10 veces mayor que un vídeo convencional en el mismo canal, mientras que los comentarios y reacciones son nueve veces más amplias.

- GTLive: canal sobre videojuegos perteneciente a Theorist Media. Realiza emisiones en directo en YouTube desde 2015. A comienzos de 2018, este tipo de vídeos tenían visualizaciones oscilantes entre los 15 y 17 mil espectadores, pero seis meses más tarde su volumen aumentó entre los 22 y 25 mil.

- The Young Turks Network: red de video digital con contenidos informativos y de tertulia política. TYT Network afirma haber sido uno de los pioneros en utilizar YouTube Live en 2011 y el primero en hacer propia la versión móvil en 2016. Si bien no entregan datos, afirman que han tenido un crecimiento "definitivamente notable" y que los contenidos SMLS emitidos desde un dispositivo móvil tienen un promedio de visualización aún mayor.

Diversos editores de contenido y expertos de la industria coinciden en que los medios digitales se cansaron de Facebook por el formato de subsidios porque no duran más de un año promedio, y que una vez terminado ese periodo quedan en una especie de abandono. Además, con YouTube no se necesita iniciar sesión para acceder a sus contenidos.

Según el reporte previamente citado de Digiday UK, los expertos entrevistados coinciden en que YouTube Live entró a la conocida paradoja de "el huevo o lo gallina" porque como niegan cambio alguno en el algoritmo, no se sabe estas mejoras responden a un crecimiento orgánico (y espontáneo) de la audiencia en directo o se debe a un cambio no informado en su sistema de posicionamiento de contenidos.

De todos modos, la sospecha de los principales editores periodísticos es que YouTube necesita afianzarse como la principal videoteca en línea ante una posible arremetida de Twitch (Patel, 2018b) y ante una estrategia de salida de Facebook (Méndez Nieto, Rivera, \& Palomo Torres, 2018, págs. 40-41).

\subsection{YouTube TV, la llegada del SMLTV}

En trabajos anteriores (Apablaza-Campos, 2017b), analizamos las diferencias entre una emisión televisiva convencional y otra en SMLS: "el live streaming permite que la audiencia dirija la emisión a través de los mensajes que coloca bajo el vídeo" (Mark Frankel, entevistado en Shawley, 2016, p.47). Por ello, la réplica de las señales de TV en las redes sociales ha permitido la aparición del Social Media Live Television (SMLTV).

Twitter fue la pionera en emisiones en SMLTV en 2016, cuando firmó un acuerdo con la NFL para emitir un partido semanal, contenidos deportivos en exclusiva y la llegada de un canal informativo 24/7 junto a Bloomberg (Christiansen, 2017). Facebook, por su parte, no se quedó atrás y lanzó en la segunda parte de 2017 Watch (Castillo, 2018), una plataforma de contenidos que ha tenido problemas para seducir a las grandes cadenas que desconfían del ya mencionado programa de subsidios de la red social (Patel, 2018c). Facebook Watch llegó a España en agosto de 2018, funcionando como un video feed (Prieto, 2018). 


\begin{tabular}{|c|c|c|}
\hline Tipo & Canales & $\begin{array}{c}\text { Precio } \\
\text { mensual/plan }\end{array}$ \\
\hline Generalistas* & $\begin{array}{l}\text { ABC, CBS, The CW, Fox, } \\
\text { MyNetworkTV, NBC y } \\
\text { Telemundo }\end{array}$ & \multirow{6}{*}{$\begin{array}{l}40 \text { USD/ } \\
\text { básico }\end{array}$} \\
\hline $\begin{array}{l}\text { Entreteni- } \\
\text { miento }\end{array}$ & $\begin{array}{l}\text { AMC, BBC America, } \\
\text { Bravo, Cheddar, Comet, } \\
\text { Decades, iE!, Freeform, } \\
\text { FX, FX Movie Channel, } \\
\text { FXX, IFC, Pop, Sundan- } \\
\text { ceTV, Syfy, TBS, TNT, } \\
\text { Turner Classic Movies, } \\
\text { TruTV, Universo, USA } \\
\text { Network, WeTVy You- } \\
\text { Tube Red }\end{array}$ & \\
\hline Familiares & $\begin{array}{l}\text { Cartoon Network, } \\
\text { Disney Channel, Disney } \\
\text { Junior, Disney XD y Uni- } \\
\text { versal Kids }\end{array}$ & \\
\hline Informativos & $\begin{array}{l}\text { BBC World News, Big } \\
\text { Cheddar News, CNBC, } \\
\text { CNN, Fox Business } \\
\text { Network, Fox News } \\
\text { Channel, HLN, MSNBC, } \\
\text { NECN, Newsy y TYT } \\
\text { Network }\end{array}$ & \\
\hline Deportes** & $\begin{array}{l}\text { Big Ten Network, CBS } \\
\text { Sports Network, ESPN, } \\
\text { ESPN 2, ESPNews, } \\
\text { ESPNU, Fox Sports } \\
\text { Networks, FS1, FS2, Golf } \\
\text { Channel, Los Angeles FC } \\
\text { games, MLB Network, } \\
\text { NBA TV, NBCSN, } \\
\text { NBC Sports Regio- } \\
\text { nal Networks, NESN, } \\
\text { Olympic Channel, SEC } \\
\text { Network, SNY, Tennis } \\
\text { Channel y YES Network }\end{array}$ & \\
\hline Otros & $\begin{array}{l}\text { National Geographic } \\
\text { Channel, Nat Geo Wild, } \\
\text { Smithsonian Channel y } \\
\text { Tastemade }\end{array}$ & \\
\hline \multirow{4}{*}{$\begin{array}{l}\text { Complementa- } \\
\text { rios }\end{array}$} & Showtime & $\begin{array}{l}11 \text { USD/Pre- } \\
\text { mium }\end{array}$ \\
\hline & Fox Soccer Plus & $\begin{array}{l}15 \text { USD/pre- } \\
\text { mium }\end{array}$ \\
\hline & Shudder & $\begin{array}{l}5 \text { USD/ } \\
\text { Premium }\end{array}$ \\
\hline & Sundance Now & $\begin{array}{l}7 \text { USD/ } \\
\text { premium }\end{array}$ \\
\hline \multicolumn{3}{|c|}{$\begin{array}{l}\text { *Desconexiones locales no están disponibles en todos los } \\
\text { estados. } \\
\text { **Redes deportivas locales disponibles solo en algunos } \\
\text { estados según factibilidad }\end{array}$} \\
\hline
\end{tabular}

\section{Tabla 1. Oferta de canales y PVP de YouTube TV en Estados} Unidos (Wikipedia, 2018).

YouTube hizo lo suyo y desde febrero de 2017 cuenta con YouTube TV, servicio de televisión en directo disponible en todo Estados Unidos, con una suscripción de 40 dólares al mes en su plan básico.

A principios de 2018, YouTube TV alcanzó un total de 300 mil suscriptores (Sherman, 2018). Su servicio, a diferencia de otras cadenas de televisión de pago, no solo dispone de una oferta de canales en abierto, de pago o autonómicos, también han convencido a los principales canales de su plataforma abierta de pasarse al broadcasting y crear una señal de emisión 24/7 (Patel, 2018a). Ha sucedido recientemente con el portal de noticias Cheddar, el de cocina Tastemade y el ya mencionado TYT Network, como se pueden distinguir en la Tabla 1 (Perez, 2018).

Desde el mundo de los anunciantes hay expectativas sobre las opciones que puede ofrecer YouTube TV. Aunque de momento solo pueden optar a paquetes publicitarios generales, se espera que a fines de 2018 sea posible invertir en ADS por eventos y acciones específicas (Peterson, 2018c). La capacidad de interactividad que dispone la red social de Google es uno de los principales atributos que puede seducir a las marcas de invertir en contenidos broadcasting (Peterson, 2018a).

\section{Resultados}

En medio de una serie de problemáticas sociales y cuestionamientos por parte de diversos organismos internacionales se realizaron las Elecciones Presidenciales 2018 en Venezuela. La compleja situación política en el país latinoamericano claramente influyó en las decisiones editoriales de los medios digitales.

Como el principal grupo de la oposición, la Mesa de Unidad Democrática, no participó de los comicios, algunos de los cibermedios afines, como Efecto Cocuyo y NTN 24 Venezuela, estudiados en trabajos anteriores ${ }^{3}$, decidieron no realizar emisiones SMLS durante ese día, aunque sí volvieron con sus contenidos en directo habituales a partir del día siguiente. Es posible que, además, se debiera a recomendaciones de organizaciones periodísticas internacionales de no usar sus dispositivos móviles en centros electorales ante el peligro de agresiones (Nalvarte, 2018).

Sin embargo, es interesante destacar que, a diferencia de jornadas electorales pasadas, los medios públicos y los canales de televisión en abierto decidieron sumarse a las emisiones en live streaming, entregando un panorama informativo más amplio y plural que en procesos anteriores.

Teniendo en cuenta estas consideraciones, la muestra de contenidos escogidos corresponde a todos los contenidos SMLS que los canales mencionados emitieron durante el día 20 de mayo y, gracias a las estadísticas de YouTube y de herramientas como Socialblade ${ }^{4}$, se tomaron en cuenta datos como las reproducciones promedio y acumuladas ${ }^{5}$ como se puede distinguir en la Tabla 2.

De los resultados visibles en la Tabla 2, es necesario realizar las siguientes consideraciones:

- Al momento de la toma de la muestra en el horario ya mencionado, solo Venezolanos por la Información, Glo- 


\begin{tabular}{|c|c|c|c|c|c|c|c|}
\hline Medio & Canal YouTube & Inicio emisión & $\begin{array}{l}\text { Repro- } \\
\text { ducciones } \\
\text { promedio }\end{array}$ & $\begin{array}{c}\text { Repro- } \\
\text { ducciones } \\
\text { acumuladas }\end{array}$ & Comentarios & Me gusta & No me gusta \\
\hline $\begin{array}{l}\text { Venezolanos por } \\
\text { la Información }\end{array}$ & VPItv & 20/05/2018 & 20.608 & 465.372 & N/D & 2.600 & 436 \\
\hline Venevisión & $\begin{array}{l}\text { Noticiero } \\
\text { Venevisión }\end{array}$ & 16/05/2018 & 2.000 & 235.100 & N/D & 1.200 & 770 \\
\hline Globovisión & $\begin{array}{l}\text { Globovisión } \\
\text { en directo }\end{array}$ & 20/05/2018 & 532 & 159.727 & N/D & 335 & 185 \\
\hline TeleSUR & TeleSUR TV & 19/05/2018 & 897 & 147.100 & 314 & 1.300 & 440 \\
\hline Televen & Televen EN VIVO & 15/05/2018 & 792 & 122.600 & N/D & 525 & 267 \\
\hline $\begin{array}{l}\text { Venezolana } \\
\text { de Televisión }\end{array}$ & $\begin{array}{l}\text { VTV Voto } 2018 \text { - } \\
\text { El pueblo decide }\end{array}$ & 20/05/2018 & 80 & 4.300 & 1 & 49 & 31 \\
\hline \multicolumn{3}{|r|}{ Promedio } & 4.152 & 189.033 & 158 & 1.002 & 355 \\
\hline \multicolumn{3}{|r|}{ Acumulado } & 24.909 & 1.134 .199 & 315 & 6.009 & 2.129 \\
\hline
\end{tabular}

Tabla 2. Rendimiento e interacciones de medios venezolanos en YouTube Live durante las Elecciones Presidenciales. Fuente: elaboración propia.

bovisión y Venezolana de Televisión habían concluido sus emisiones.

- Globovisión fue el único medio que, una vez concluida su emisión, almacenó su contenido en la sección Vídeos. Su duración fue de 3 horas 18 minutos y 29 segundos.

- En la columna de Comentarios, correspondiente a los mensajes publicados en la sección "chat en vivo", no hay datos en 4 de los 6 medios estudiados porque los emisores eligieron desactivar la opción.

- En cuanto al contenido, en todos los medios estudiados se repite la fórmula: réplica de sus emisiones televisivas. Mismo caso de Venezolanos por la Información que, pese a no disponer de una señal televisiva en abierto, realiza emisiones en directo 24/7 en YouTube. Aún así, durante el 20 de mayo realizó una emisión especial que solo duró un día.

- En total, los medios venezolanos recibieron 8.138 reacciones ("Me gusta" y "No me gusta") en sus emisiones de YouTube Live. Un 74\% de esos emoticonos son positivos. Venezolanos por la Información fue el medio que tuvo un porcentaje mayor de valoraciones positivas (86\%) y Venezolana de Televisión, el menor (61\%).

- Es importante destacar que, de acuerdo a nuestro seguimiento de las publicaciones SMLS, los medios que comenzaron su emisión en directo días antes de las elecciones no generaron demasiado contenido previo. Es decir, si bien estaban emitiendo de forma continua, muchos de ellos (a excepción de TeleSUR) usaron cartas de ajustes o simplemente la pantalla en negro durante gran parte de sus respectivos directos.

- En resumen, la estrategia de vídeo en directo de medios televisivos venezolanos fue la réplica de sus informativos en SMLS para ampliar su alcance convencional en abierto. Por ello, no es de sorprender que los mejores resultados correspondan a Venezolanos por la Información porque es un medio nativo digital y emitió en un formato de mayor calidad (HD y 4K).

\section{Conclusiones}

1. El Social Media Live Streaming (SMLS) ha madurado en un entorno en el cual, por diversos factores, el consumo de vídeos en línea está en constante crecimiento. Tanto la producción y reproducción a través de las redes sociales como la entrega de feedback al emisor de las reacciones sus audiencias son las principales claves que lo explican.

2. De acuerdo a las definiciones de redes sociales más consensuadas por diversos académicos y expertos en entornos digitales es posible decir que YouTube es una red social porque sus usuarios -ya sean creadores o espectadores- pueden establecer conexiones, relaciones, comunicación, crear comunidades y compartir contenido.

3. En línea con el punto anterior se puede considerar a YouTube Live, denominación de los vídeos en directo de la red social de Google, como una tecnología SMLS por cumplir con las principales claves mencionadas al principio de este apartado.

4. Actualmente, es posible clasificar el SMLS en tres tipos: redes sociales propias, funcionalidades para perfiles, y aplicaciones externas. Tras una década desde su primera emisión en directo, YouTube ha realizado experimentos con las tres; pero finalmente ha privilegiado la funcionalidad para sus canales con modalidades para aficionados (YouTube Live) y expertos (YouTube Live Events).

5. En 2017, Facebook Live era la tecnología SMLS más usada, 
pero tras su cambio de algoritmo y la filtración de datos de sus perfiles, diversos editores han cuestionado sus impactos. Los cibermedios han encontrado el atractivo de emitir en directo en otras redes sociales como Twitch y el propio YouTube.

6. Diversos canales de medios estadounidenses, con años de experiencia en YouTube Live, reconocen que este 2018 han tenido una importante subida de su audiencia e interacciones. Algunos de ellos, también, han concluido que un live streaming desde un dispositivo móvil tiene un rendimiento superior al promedio de publicaciones.

7. YouTube ha experimentado en Social Media Live Television (SMLTV) con la creación de un servicio de televisión en directo de pago llamado YouTube TV. Hay expectativas de los anunciantes de que pueda generar mensajes interactivos para la audiencia en contenidos broadcasting.

8. Medios televisivos venezolanos (de broadcasting y de streaming) han realizado emisiones SMLS en YouTube Live durante las Elecciones Presidenciales 2018. La muestra, de seis medios estudiados, arroja una audiencia acumulada superior al millón de reproducciones y un cerca de 8.500 interacciones (comentarios, "Me gusta" y "No me gusta").

9. En resumen, creemos que YouTube Live es una tecnología de Social Media Live Streaming que permite al periodismo ampliar la visibilidad de sus contenidos audiovisuales, mejorando sus niveles de interacción y de engagement con sus audiencias en comparación con un vídeo convencional en redes sociales. Además, sus resultados en continuo crecimiento han llevado a diversos cibermedios a dar más relevancia a este tipo de contenidos dentro de sus respectivas estrategias digitales.

\section{Agradecimientos}

Esta investigación cuenta con el apoyo de CONICYT (Comisión Nacional Científica y Tecnológica de Chile), a través del programa "Becas Chile" Doctorado en el Extranjero, número de folio 72170334 correspondiente a Alexis Apablaza-Campos.

Asimismo, este trabajo forma parte del proyecto "Creación y contenido interactivo en la comunicación de información audiovisual: audiencias, diseño, sistemas y formatos". Ref: CS02015-64955-C4-2-R (MINECO/FEDER), Ministerio de Economía y Competitividad (España).

\section{Notas a final de página}

1. Web de vídeos en directo que operó durante 2007 y 2014. Fue absorbida por Twitch, su compañía subsidiaria, previo a su venta a Amazon.
2. Primera app móvil de vídeos en directo que generaba contenido a través de perfiles en redes sociales de los usuarios. Solo duró 20 meses entre 2015 y 2016 (Mlot, 2016).

3. En investigaciones anteriores, hemos realizado estudios de casos de coberturas SMLS de cibermedios a través de Periscope y Facebook durante las siguientes elecciones venezolanas de 2017: Consulta Nacional, Asamblea Nacional Constituyente y votaciones Regionales.

4. Herramienta de analítica de redes sociales disponible en https:// socialblade.com/

5. Datos extraídos a las 23:59 (hora española) del lunes 21 de mayo de 2018.

\section{Referencias}

Adveischool (30 de septiembre de 2016a). YouTube Community la Red Social Propia de YouTube. Adveischool Recuperado de https://adveischool.com/youtube-community-2

Adveischool. (1 de septiembre de 2016b). Tutorial de YouTube Live: Cómo Emitir Video en Directo. Adveischool. Recuperado de https:// adveischool.com/tutorial-de-youtube-live/

Alexa. (2018). Top 500 Global Sites. Alexa (4/06/2018), Recuperado de https://www.alexa.com/topsites

Apablaza-Campos, A. (2017a). Marketing de Contenidos: oportunidades para medios digitales. XXVII Encuentro de Diarios Regionales (pág. 33). Valparaíso: ANP Chile.

Apablaza-Campos, A. (2017b). Social Media Live Streaming (SMLS) para medios digitales. Congreso Internacional de Investigación en Comunicación. Girona: Societat Catalana de Comunicació.

Apablaza-Campos, A. (2017c). Social media live streaming y su impacto en los medios de comunicación: el caso de Periscope. IV Congreso Nacional de la Asociación Chilena de Investigadores en Comunicación. Santiago de Chile: INCOM.

Argila, J. (2017). How to create video news that rocks in social media. University of Oxford. Oxford: Reuters Institute. Recuperado de https://reutersinstitute.politics.ox.ac.uk/sites/default/ files/2017-09/Argila\%2C\%2OHow\%20to\%20create\%20video\%20 news\%20that\%20rocks\%20in\%20social\%20media.pdf

BBC News Mundo. (2 de junio de 2018). Las 3 redes sociales favoritas de los adolescentes de Estados Unidos (y ninguna es Facebook). BBC News Mundo. Recuperado de http://www.bbc.com/mundo/noticias-44338587

Boyd, D. M., y Ellison, N. B. (2007). Social Network Sites: Definition, History, and Scholarship. Journal of Computer-Mediated Communication, 21. DOI: 10.1111/j.1083-6101.2007.00393.x

Brown, P. (23 de febrero de 2018). RIP Facebook Live: As subsidies end, so does publisher participation. Columbia Journalism Review. Recuperado de https://www.cjr.org/tow_center/facebook-live-overas-payments-end.php

Castillo, M. (1 de febrero de 2017). Mark Zuckerberg sees video as a 'mega trend' and is gunning for YouTube. CNBC. Recuperado de https://www.cnbc.com/2017/02/01/mark-zuckerberg-video-mega-trend-like-mobile.html

Castillo, M. (5 de febrero de 2018). Facebook is planning a more direct assault on YouTube, sources say. CNBC. Recuperado de https://www. cnbc.com/2018/02/05/facebook-wants-to-open-up-its-videos-platform-to-more-online-influencers-and-advertisers-.html

Chowdhry, A. (26 de febrero de 2018). YouTube Live Gains Several 
New Features. Forbes. Recuperado de https://www.forbes.com/ sites/amitchowdhry/2018/02/26/youtube-live-new-features/\#7367c085687f

Christiansen, A. (2 de mayo de 2017). Twitter recurre a la TV y anuncia 12 shows para mantenerse vigente. La Tercera. Recuperado de http://www2.latercera.com/noticia/twitter-recurre-la-tv-anuncia-12-shows-mantenerse-vigente/

Coldewey, D. (2 de junio de 2018). It's OK to leave Facebook. TechCrunch. Recuperado de https://techcrunch.com/2018/06/02/ its-ok-to-leave-facebook/

Constine, J. (6 de abril de 2017). One in five Facebook videos is Live as it seizes the verb. Techcrunch. Recuperado de https://techcrunch. com/2017/04/06/live-video/

Dobuzinskis, A. (12 de noviembre de 2008). YouTube ventures into live event webcasting. Reuters. Recuperado de https://www.reuters. com/article/us-media-youtube-idUSTRE4AB40U20081112

Doctor, K. (2010). Newsonomics: Twelve New Trends That Will Shape the News You Get. New York: St. Martin's Press.

El Economista América. (5 de enero de 2016). Periscope: la aplicación estrella del año 2015. El Economista. Recuperado de http://www.eleconomista.es/telecomunicacion-tecnologia-cl/ noticias/7259884/01/16/-Periscope-la-aplicacion-estrella-del-ano-2015.html

Gomis, L. (1991). Teoría del periodismo: cómo se forma el presente. Barcelona: Paidós.

Gross, D. (13 de septiembre de 2010). YouTube testing live streaming. CNN. Recuperado de http://edition.cnn.com/2010/TECH/web/09/13/ youtube.livestreaming/index.html?hpt=C2

IAB Spain. (2010). II Estudio sobre Redes Sociales en Internet. Madrid: Elogia Marketing Sigular.

Kalogeropoulos, A., Cherubini, F., y Newman, N. (2016). Digital News Proyect: The future of online news video. University of Oxford. Oxford: Reuters Institute. Recuperado de https://reutersinstitute. politics.ox.ac.uk/sites/default/files/research/files/The\%2520Future\%2520of\%25200nline\%2520News\%2520Video.pdf

MacNN. (13 de diciembre de 2013). YouTube opens live streaming for all verified accounts. Macnn. Recuperado de http://www.macnn. com/articles/13/12/13/youtube.abandons.100.subscriber.limit.for. live.streaming

McCue, T. (22 de septiembre de 2017). Top 10 Video Marketing Trends And Statistics Roundup 2017. Forbes. Recuperado de https://www. forbes.com/sites/tjmccue/2017/09/22/top-10-video-marketingtrends-and-statistics-roundup-2017/\#73ed93177103

Méndez Nieto, A., Rivera, A., y Palomo Torres, M. (Mayo de 2018). Facebook: ¿enemigo o aliado? Las empresas periodísticas españolas valoran su relación con la principal red social del mundo. Hipertext. net, (16), 32-42. D0I:10.31009/hipertext.net.2018.i16.07

Mlot, S. (3 de octubre de 2016). Streaming App Meerkat Is Officially Dead. PC Mag. Recuperado de https://www.pcmag.com/ news/348393/streaming-app-meerkat-is-officially-dead

Nación. (26 de noviembre de 2006). 'Time' escoge a You Tube como el invento del 2006. Nación. Recuperado de http://www.nacion.com/ vivir/Time-escoge-You-Tube-invento_0_869313124.html

Nalvarte, P. (23 de mayo de 2018). Prensa venezolana sufre agresiones y restricciones de cobertura en centros de votación durante elecciones presidenciales. Knight Center for the Journalism in the
Americas. Recuperado de https://knightcenter.utexas.edu/es/ blog/00-19685-prensa-venezolana-sufre-agresiones-y-restricciones-de-cobertura-en-centros-de-votacion

Ong, T. (21 de marzo de 2018). YouTube live-streaming gets easier from computers and phones. The Verge. Recuperado de https:// www.theverge.com/2018/3/21/17146288/youtube-live-streaming-tool-chrome-browsers-camera-app-android

ONTSI. (2011). Las Redes Sociales en Internet. Madrid: Observatorio Nacional de las Telecomunicaciones y de la SI.

Palacios, M., y Díaz Noci, J. (Edits.). (2009). Online journalism : Research methods. A multidisciplinary approach in comparative perspective. Salvador de Bahía: Argitalpen Zerbitzua.

Parker, L. (12 de abril de 2017). A Chat With a Live Streamer Is Yours, for a Price. The New York Times. Recuperado de https://www. nytimes.com/2017/04/12/technology/personaltech/paying-for-livestream-chat.html

Patel, S. (6 de abril de 2018a). YouTube TV eyes new linear channels from digital publishers. Digiday UK. Recuperado de https://digiday. com/media/youtube-tv-eyes-new-linear-channels-from-digitalpublishers

Patel, S. (17 de mayo de 2018b). Magazine publishers with video ambitions see YouTube as safer bet than Facebook. Digiday UK. Recuperado de https://digiday.com/media/reliable-smaller-video-publishers-see-youtube-safe-harbor

Patel, S. (9 de mayo de 2018c). Publishers are wrestling with Facebook's desire to own Facebook-funded Watch shows. Digiday UK. Recuperado de https://digiday.com/media/publishers-are-wrestling-with-facebooks-desire-to-own-facebook-funded-watch-shows

Perez, S. (18 de abril de 2018). YouTube TV adds its first digital-only networks with launch of two channels from Cheddar. TechCrunch. Recuperado de https://techcrunch.com/2018/04/18/youtube-tvadds-its-first-digital-only-networks-with-launch-of-two-channelsfrom-cheddar

Perlberg, S., y Sheetharaman, D. (22 de junio de 2016). Facebook Signs Deals With Media Companies, Celebrities for Facebook Live. The Wall Street Journal. Recuperado de https://www.wsj.com/articles/ facebook-signs-deals-with-media-companies-celebrities-for-facebook-live-1466533472

Peterson, T. (9 de abril de 2018a). Ad buyers expect Hulu, YouTube to start selling ads in their live TV services. Digiday UK. Recuperado de https://digiday.com/media/ad-buyers-expect-hulu-youtube-start-selling-ads-live-tv-services/?utm_campaign=digidaydisctutm_source=twitter\&utm_medium=social

Peterson, T. (16 de mayo de 2018b). YouTube channels are seeing a lift in live video viewership. Digiday UK. Recuperado de https://digiday. com/media/youtube-channels-seeing-lift-live-video-viewershi$\mathrm{p} /$ ?utm_source=twitter\&utm_medium=social\&utm_campaign=digidaydis

Peterson, T. (5 de junio de 2018c). YouTube TV's digital channels experiment with different ad loads while they still can. Digiday UK. Recuperado de https://digiday.com/media/youtube-tvs-digital-channels-experiment-different-ad-loads-still-can/

Prieto, M. (30 de agosto de 2018). Facebook lanza en España su plataforma de vídeo Watch. Expansión. Recuperado de http://www. expansion.com/economia-digital/companias/2018/08/29/5b86bea9e5fdea2d458b4623.html

PuroMarketing. (8 de mayo de 2018). La cuarta parte de la población 
mundial ya usa YouTube. Puro Marketing. Recuperado de https:// puromarketing.com/15/30319/cuarta-parte-poblacion-mundial-usa-youtube.html

Scolari, C. (Ed.). (2015). Ecología de los Medios: Entornos, evoluciones e interpretaciones. Barcelona: Gedisa.

Shawley, P.. (2016). A fresh face for storytelling. Television, 53(9), 46-47. Recuperado de https://rts.org.uk/sites/default/files/new_ television-oct16-lo-res.pdf

Sherman, A. (22 de enero de 2018). In their battle against big cable, YouTube TV and Hulu with Live TV have signed up hundreds of thousands of subscribers. CNBC. Recuperado de https://www.cnbc. com/2018/01/22/youtube-live-has-about-300k-subscribers-andhulus-live-has-450k-sources-reveal.html

Smith, A., y Anderson, M. (1 de marzo de 2018). Social Media Use in 2018. Pew Research Center. Recuperado de http://www.pewinternet. org/2018/03/01/social-media-use-in-2018

Southern, L. (21 de mayo de 2018). With Facebook Live views falling, BuzzFeed looks to Twitch. Digiday UK. Recuperado de https://digiday. com/media/facebook-live-views-falling-buzzfeed-looks-twitch/?utm_campaign=digidaydis\&utm_medium=social\&utm_source=twitter

Stewart, D. R., y Littau, J. (2016). Up, Periscope: Mobile Streaming Video Technologies, Privacy in Public, and the Right to Record. Journalism \& Mass Communication Quarterly, 93 (2), 312-323.

Villas-Boas, A. (7 de marzo de 2018). What is $5 G$ and how fast will it be? The Independent. Recuperado de https://www.independent. co.uk/life-style/gadgets-and-tech/5g-phone-internet-4g-howfast-when-iphone-android-mobile-a8244411.html

Wikipedia. (2018). YouTube TV. Recuperado de https://en.wikipedia. org/wiki/YouTube_TV

Yeung, K. (23 de marzo de 2016). Google is building YouTube Connect, a livestreaming app to take on Periscope. VentureBeat. Recuperado de https://venturebeat.com/2016/03/23/google-is-building-youtube-connect-a-live-streaming-app-to-take-on-periscope

Yin, R. K. (2018). Case Study Research and Applications: Design and
Methods (Sexta ed.). Los Angeles: SAGE Publications.

YouTube. (8 de abril de 2011). YouTube is going LIVE. YouTube Official Blog. Recuperado de https://youtube.googleblog.com/2011/04/ youtube-is-going-live.html

YouTube. (2018a). Prensa (27 de mayo de 2018). Recuperado de https://www.youtube.com/intl/es-419/yt/about/press/

YouTube. (2018b). YouTube Live (3 de junio de 2018). Recuperado de https://www.youtube.com/live_dashboard_splash

YouTube. (2018c). YouTube Live Events (3 de junio de 2018). Recuperado de https://www.youtube.com/my_live_events

Zuckerberg, M. (12 de enero de 2018). One of our big focus areas for 2018 is making... Post. Facebook notes. Recuperado de https://www. facebook.com/zuck/posts/10104413015393571

\section{CV}

Alexis Apablaza-Campos. Periodista y Licenciado en Comunicación Social por la Universidad Andrés Bello (Chile). Máster en Comunicación Empresarial por el Instituto de Formación Continua-IL3 de la Universidad de Barcelona y Magíster en Comunicación Corporativa y Responsabilidad Social Empresarial por la Universidad Andrés Bello (Chile). Investigador del Programa de Doctorado del Departamento de Comunicación de la Universitat Pompeu Fabra. Colaborador del Grupo de Investigación DigiDoc y editor/analista del Observatorio de Cibermedios (UPF). Consultor en Comunicación Digital y columnista editorial Diario El Rancagüino. Profesor Asociado de la Escuela de Periodismo y Relaciones Públicas Corporativas de la Universidad UNIACC (Chile) y profesor invitado ESIC Business \& Marketing School Barcelona. https://smls.info/

\section{Observatorio} de Cibermedios

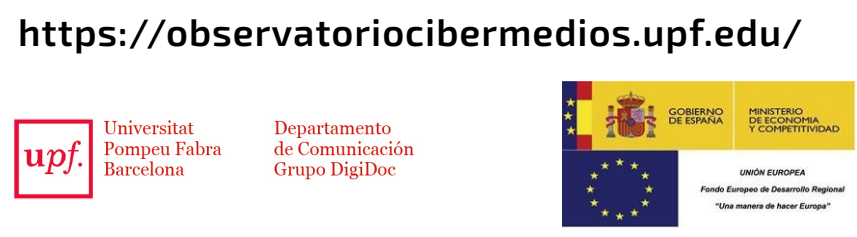

El Observatorio de Cibermedios es una producción del Grupo de Investigación en Documentación Digital y Comunicación Interactiva (DigiDoc) del Departamento de Comunicación de la Universitat Pompeu Fabra.

El Observatorio de Cibermedios (OCM) forma parte del proyecto del Plan Nacional "Creación y contenido interactivo en la comunicación de información audiovisual: audiencias, diseño, sistemas y formatos". CS02015-64955-C4-2-R (MINECO/ FEDER), Ministerio de Economía y Competitividad (España). 